\title{
Paeoniflorin inhibits cell viability and invasion of liver cancer cells via inhibition of Skp2
}

\author{
HONG LIU ${ }^{1}$, LILI ZANG ${ }^{2}$, JUN ZHAO ${ }^{2}$, ZHAOLIN WANG $^{3}$ and LINGYUN LI ${ }^{2}$ \\ ${ }^{1}$ Department of Liver Disease, Yantai Infectious Diseases Hospital, Yantai, Shangdong 264001; \\ ${ }^{2}$ Department of Gastroenterology, Yantai Yu Huang Ding Hospital, Yantai, Shangdong 264003; \\ ${ }^{3}$ Department of Information and Electrical Engineering, Ludong University, Yantai, Shangdong 264025, P.R. China
}

Received October 9, 2018; Accepted December 3, 2019

DOI: $10.3892 / \mathrm{ol} .2020 .11424$

\begin{abstract}
Paeoniflorin (PF) has been demonstrated to exert tumor suppressive functions in various types of human cancer. However, the mechanisms of PF-mediated anti-tumor activity have not been fully elucidated. S-phase kinase associated protein 2 ( $\mathrm{Skp} 2$ ) has been characterized as an oncoprotein that contributes to carcinogenesis. Therefore, the inhibition of Skp2 may be a useful approach for the treatment of various types of human cancer. The present study explored whether PF inhibited the expression of Skp2 in liver cancer cells, leading to cell viability inhibition, induction of apoptosis, and suppression of migration and invasion. PF treatment led to inhibition of Skp2 expression in liver cancer cells. The overexpression of Skp2 abolished PF-mediated anti-cancer activity, whereas the downregulation of Skp2 enhanced this type of activity. The data indicated that PF may be considered as a novel inhibitor of Skp2 in liver cancer cells.
\end{abstract}

\section{Introduction}

Liver cancer is common malignancy with poor prognosis. In the United States of America (USA), 42,220 individuals were diagnosed with liver cancer in 2018, resulting in 30,200 fatalities (1). In addition, the 5-year relative survival rate for liver cancer is $\sim 18 \%$ in the USA (1). Due to recent advances in liver transplantation technologies, patients with liver cancer often demonstrate optimal survival rates if the cancer is diagnosed at a localized stage (2). However, the survival rates of patients with liver cancer with distant tumor localization remains low (2). Therefore, it is necessary to identify novel agents that may improve the treatment of patients with liver cancer.

Correspondence to: Dr Lingyun Li, Department of Gastroenterology, Yantai Yu Huang Ding Hospital, 1181 Harbor City East Main Street, Yantai, Shandong 264003, P.R. China

E-mail: lingyunli2018@126.com

Key words: paeoniflorin, viability, S-phase kinase associated protein 2 , liver cancer, cell cycle, invasion
S-phase kinase associated protein 2 (Skp2) has been demonstrated to control cellular growth, tumor development and progression (3). Skp2 is a component of the Skp1-Cul1-F-box protein E3 ubiquitin ligase that mediates the ubiquitination and subsequent proteasomal degradation of targeted substrates (4). Skp2 has been characterized as an oncoprotein, and functions by facilitating the deregulated ubiquitination and proteolysis of several tumor suppressor proteins (4). Skp2 has been demonstrated to enhance tumor progression and development via the regulation of various biological processes, namely cell proliferation, the cell cycle, apoptosis, invasion and metastasis (4-6). In liver cancer, the expression of Skp2 is associated with histological grade and tumor size, suggesting that Skp2 may be a prognostic biomarker for patients with liver cancer $(7,8)$. A recent study demonstrated that $\mathrm{Skp} 2$ promoted the proliferation, colony formation, migration and invasion of liver cancer cells (8). Therefore, Skp2 may be a potential target in the treatment of liver cancer.

Paeoniflorin (PF) is a bioactive component isolated from the paeony root, which exerts multiple immunoregulatory, anti-hyperglycemic and anti-hypotensive effects $(9,10)$. PF has been suggested to possess anti-tumor functions in a variety of human cancer types, including liver cancer (11-15). However, PF did not affect normal hepatocyte growth, and was demonstrated to induce apoptosis via endoplasmic reticulum (ER) stress and activation of the mitochondria-dependent pathway (16). However, the mechanisms of PF-mediated tumor suppressive function have not been fully identified. In the present study, PF-induced anti-tumor activity was investigated in liver cancer cells. As Skp2 is an important oncoprotein in liver cancer cells, whether PF inhibited Skp2 expression was determined. It was identified that PF inhibited the expression levels of Skp2 in liver cancer cells. The data suggest that inhibition of Skp2 by PF may be a novel therapeutic approach for treating patients with liver cancer.

\section{Materials and methods}

Reagents. PF was purchased from the HuanYu Biotechnology Development Company. MTT was purchased from Sigma-Aldrich; Merck KGaA. The Annexin apoptosis assay kit was obtained from Beyotime Institute of Biotechnology. The Transwell chambers and Matrigel were purchased from 
BD Biosciences. Lipofectamine ${ }^{\circledR}$ 2,000 reagent was obtained from Invitrogen; Thermo Fisher Scientific, Inc. The anti-Skp2 (1:2,000; cat. no. 4358), anti-tubulin (1:5,000; cat. no. 2146), anti- $\beta$-actin (1:5,000; cat. no. 3700), anti-cyclin dependent kinase inhibitor 1A (p21; 1:1,000; cat. no. 2491) and anti-cyclin dependent kinase inhibitor 1C (p57; 1:1,000; cat. no. 2557) antibodies were obtained from Cell Signaling Technology, Inc. Goat anti-mouse secondary antibody (1:5,000; cat. no. A-11031) and goat anti-rabbit secondary antibody (1:5,000; cat. no. A-11034) were purchased from Thermo Fisher Scientific, Inc.

Cell culture. The human liver cancer HepG2 cell line was purchased from The Cell Bank of Type Culture Collection of Shanghai Chinese Academy of Sciences. HepG2 cells were grown in Dulbecco's modified eagle's medium (DMEM; Invitrogen; Thermo Fisher Scientific, Inc.) supplemented with 10\% FBS (Thermo Fisher Scientific, Inc). The cells were cultured under a humidified atmosphere of $5 \% \mathrm{CO}_{2}$ at $37^{\circ} \mathrm{C}$.

MTT assay. The HepG2 cells were seeded into 96-well plates $\left(5 \times 10^{3}\right.$ cells/well) and incubated overnight at $37^{\circ} \mathrm{C}$ overnight. $\mathrm{PF}$ was dissolved in dimethyl sulfoxide (DMSO) and the cells were exposed to different concentrations of PF $(0,25$ and $50 \mu \mathrm{M}$ ) or $0.1 \%$ DMSO (control group) for $72 \mathrm{~h}$. The MTT assay was performed as previously described (17).

Cell apoptosis assay. The HepG2 cells $\left(5 \times 10^{5}\right.$ cells/well) were seeded into 6-well plates and exposed to $25 \mu \mathrm{M}$ PF for $48 \mathrm{~h}$. Subsequently, the cells were collected and resuspended in buffer including $5 \mu \mathrm{l}$ Annexin V-fluorescein isothiocyanate (FITC) and $5 \mu \mathrm{l}$ propidium iodide (PI) for $15 \mathrm{~min}$ at room temperature in the dark. The levels of apoptosis were then determined as previously described (17).

Wound healing assay. The HepG2 cells ( $5 \times 10^{5}$ cells/well) were cultured in DMEM supplemented with $10 \% \mathrm{FBS}$ at $37^{\circ} \mathrm{C}$ in $5 \%$ $\mathrm{CO}_{2}$ to $90 \%$ confluence. A small sterile pipette tip was used to create a rectangular lesion. Following treatment of the cells with $25 \mu \mathrm{M}$ of $\mathrm{PF}$ for $20 \mathrm{~h}$ at $37^{\circ} \mathrm{C}$, optical microscopy images were captured using an inverted light microscope (Olympus Corporation; magnification, x100).

Transwell invasion assay. The HepG2 cells were treated with $25 \mu \mathrm{M}$ of PF and cultured in the upper chambers of inserts with Matrigel using serum-free medium at $37^{\circ} \mathrm{C}$ for $20 \mathrm{~h}$. The bottom chambers were filled with complete medium. Following incubation at $37^{\circ} \mathrm{C}$ for $20 \mathrm{~h}$, the upper cells in the chambers were cleaned and the bottom cells were stained with $4 \mu \mathrm{g} / \mathrm{ml}$ of Calcein $\mathrm{AM}$ at $37^{\circ} \mathrm{C}$ for $1 \mathrm{~h}$. The cells were then analyzed by fluorescence microscopy (magnification, x100).

Reverse transcription-quantitative (RT-q)PCR analysis. Total RNA was extracted using TRIzol ${ }^{\circledR}$ reagent (Thermo Fisher Scientific, Inc.) and reverse transcribed into cDNA using TaqMan ${ }^{\circledR}$ Reverse Transcription Reagents (Thermo Fisher Scientific, Inc.). RT-qPCR was performed using Power SYBR Green PCR Master mix (Thermo Fisher Scientific, Inc.). Specifically, $2 \mu \mathrm{g}$ of total RNA was subjected to first strand cDNA synthesis in a total volume of $20 \mu \mathrm{l}$. The RT reaction was performed as follows: $37^{\circ} \mathrm{C}$ for $1 \mathrm{~h}$, followed by $95^{\circ} \mathrm{C}$ for $5 \mathrm{~min}$. The following primer sequences were used for qPCR: Skp2 forward, 5'-TGCTAAGCAGCTGTTCCAGA-3' and reverse, 5'-AAGATTCAGCTGGGTGATGG-3' and GAPDH forward, 5'-ACCCAGAAGACTGTGGATGG-3' and reverse, 5'-CAGTGAGCTTCCCGTTCAG-3'. The following thermocycling conditions were used for qPCR: Initial denaturation at $95^{\circ} \mathrm{C}$ for $10 \mathrm{~min}$; 45 cycles of $95^{\circ} \mathrm{C}$ for $15 \mathrm{sec}, 60^{\circ} \mathrm{C}$ for $1 \mathrm{~min}$; and a final extension at $72^{\circ} \mathrm{C}$ for $5 \mathrm{~min}$. Relative expression levels were quantified using the $2^{-\Delta \Delta \mathrm{Cq}}$ method as previously described $(18,19)$, and normalized to the internal reference gene GAPDH.

Western blot analysis. The cell lysates were extracted using RIPA buffer (Cell Signaling Technology, Inc.) and western blot analysis was performed as previously described (20). Image J software (version 1.52k; National Institutes of Health) was used densitometric quantification of the protein bands.

Transfection. HepG2 cells $\left(5 \times 10^{5}\right.$ cells/well) were cultured in 6 -well plates at $37^{\circ} \mathrm{C}$. The cells were either treated with $25 \mu \mathrm{M}$ of $\mathrm{PF}$, or transfected with $1 \mathrm{mg}$ of Skp2 cDNA constructor or $10 \mathrm{nM}$ of Skp2 small interfering (si)RNA (both from Shanghai Genechem Co, Ltd.) for 48 h using Lipofectamine ${ }^{\circledR} 2000$, as previously described (21). The Skp2 siRNA sequences used were as follows: Sense, 5'-CCUAUCGAACUCAGUUAUATT-3' and antisense, 5'-UAUAACUGAGUUCGAUAGGTC-3'.

Statistical analysis. Statistical analysis was performed using GraphPad Prism software (version 5.0; GraphPad Software, Inc.). The results are presented as the mean \pm standard error of the mean. The difference between groups was analyzed using analysis of variance followed by Tukey's post hoc test. $\mathrm{P}<0.05$ was considered to indicate a statistically significant difference.

\section{Results}

PF inhibits Skp2 expression in liver cancer cells. Skp2 serves an important role as an oncoprotein in liver cancer. The potential inhibitory effect of PF on Skp2 expression in liver cancer cells was investigated. RT-qPCR was performed to quantify the mRNA levels of Skp2 in HepG2 cells. It was revealed that Skp2 mRNA expression was significantly inhibited by PF treatment (Fig. 1A). The protein expression levels of Skp2 in PF-treated HepG2 cells were measured using western blot analysis. PF treatment was demonstrated to inhibit the expression of Skp2 protein in HepG2 cells (Fig. 1B and C). The downregulation of Skp2 expression by PF was evaluated by measuring the expression levels of downstream genes of Skp2, namely p21 and p57 in HepG2 cells. The western blot analysis data indicated that p57 and p21 expression levels were increased following PF treatment (Fig. 1B and C). The present data indicated that PF treatment inhibited the expression of Skp2 in liver cancer cells.

Overexpression of Skp2 rescues PF-induced cell viability inhibition and apoptosis. To further determine whether Skp2 is involved in PF-mediated tumor growth inhibition, HepG2 cells were transfected with Skp2 cDNA to overexpress Skp2, and were further treated with PF. The MTT assay was used to measure the proliferation rate of HepG2 cells following PF treatment, and transfection with the Skp2 cDNA vector. It 
A

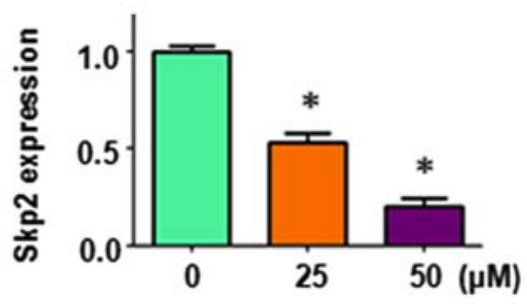

B

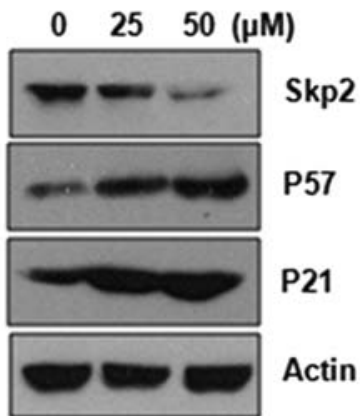

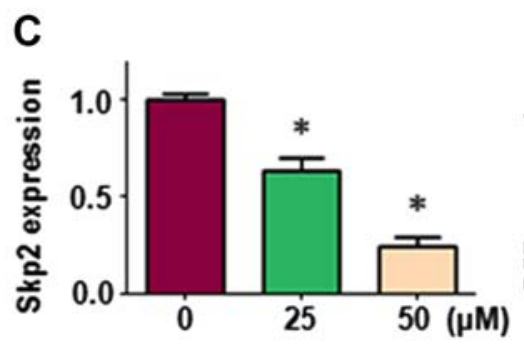
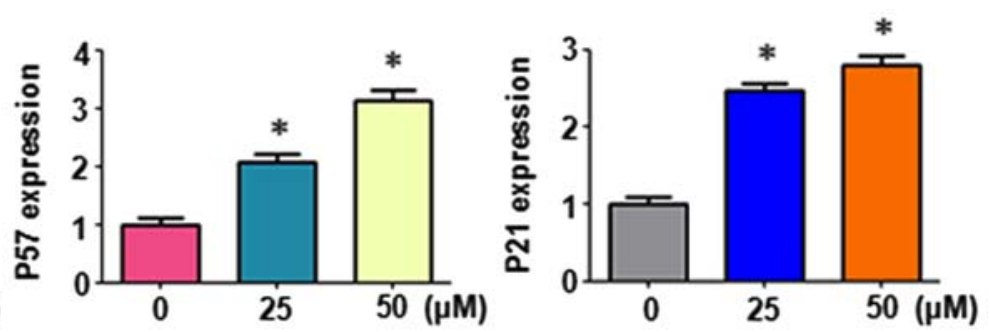

Figure 1.PF suppresses Skp2 expression. (A) The mRNA expression levels of Skp2 were measured by reverse transcription quantitative polymerase chain reaction in HepG2 cells following PF treatment. (B) Expression levels of Skp2 and its downstream targets, p21 and p57, were measured by western blot analysis in PF-treated HepG2 Cells. (C) Densitometric analyses of the western blot bands. "P $<0.05$ vs. control. PF, paeoniflorin; Skp2, S-phase kinase associated protein 2.
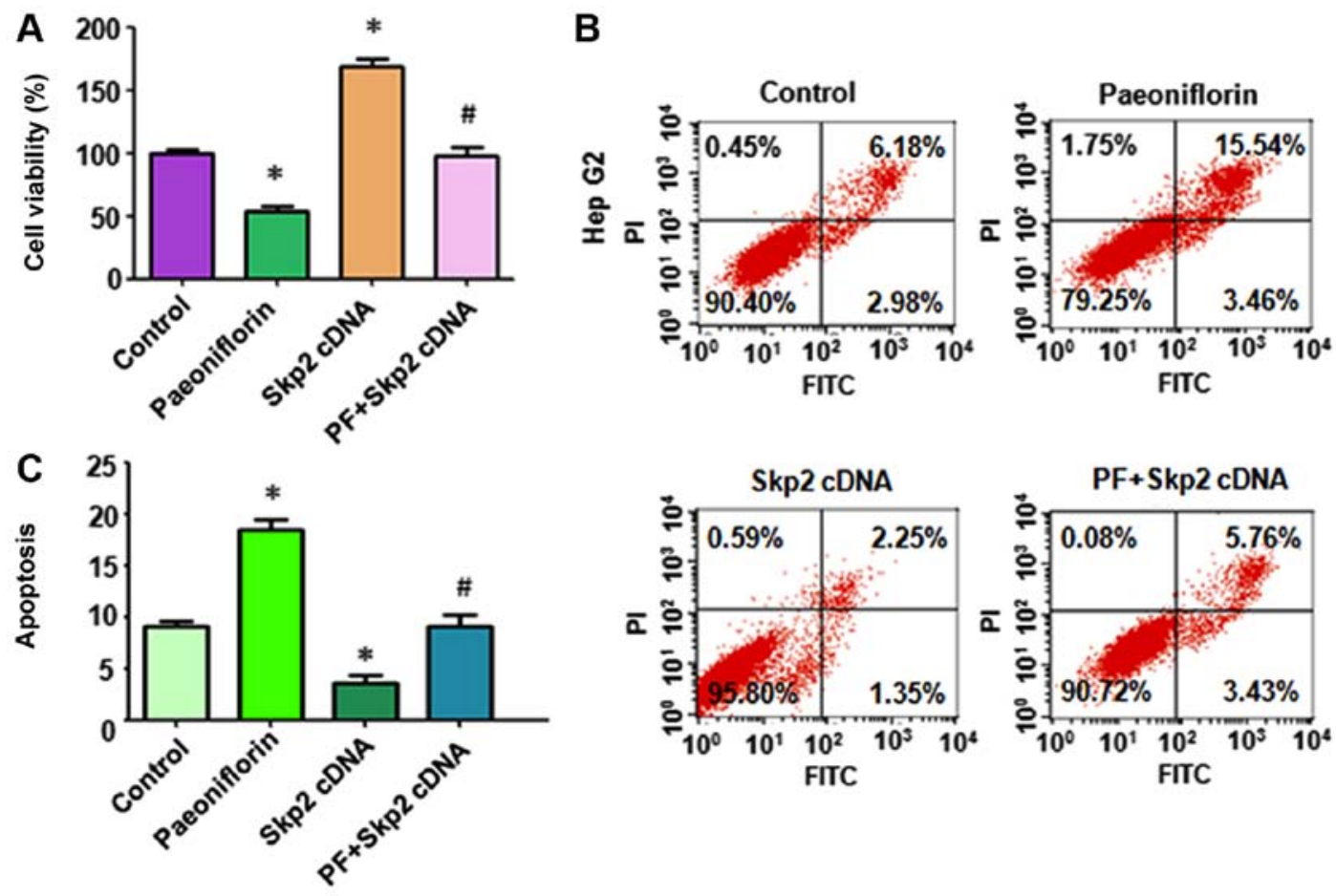

Figure 2. Overexpression of Skp2 abrogates PF-induced cell viability and apoptosis. (A) Cell viability was measured using an MTT assay following Skp2 overexpression in combination with PF treatment. (B) The number of apoptotic cells was measured by flow cytometry following Skp2 overexpression in combination with PF treatment. (C) Densitometric analyses of the western blot bands. ${ }^{*} \mathrm{P}<0.05$ vs. control; " $\mathrm{P}<0.05$ vs. $\mathrm{PF}$ treatment and Skp2 cDNA transfection, respectively. Skp2, S-phase kinase associated protein 2; PF, paeoniflorin; p21, cyclin dependent kinase inhibitor 1A; p57, cyclin dependent kinase inhibitor 1C; FITC, fluorescein isothiocyanate; PI, propidium iodide.

was identified that $25 \mu \mathrm{M}$ PF significantly inhibited HepG2 cell viability (Fig. 2A). Overexpression of Skp2 enhanced cell viability in HepG2 cells (Fig. 2A). Notably, the overexpression of Skp2 abrogated PF-induced cell viability inhibition in liver cancer cells (Fig. 2A). Next, the induction of apoptosis was measured using the FITC/PI method in HepG2 cells following
PF treatment and transfection with Skp2 cDNA. PF exposure increased the level of apoptosis (18.0\%) in the treatment group compared with the control group (9.16\%) (Fig. 2B). The overexpression of Skp2 resulted in a decrease in the levels of apoptosis in the treatment group (3.6\%) compared with the control group (9.16\%) (Fig. 2B). Notably, the overexpression of Skp2 abolished 
A
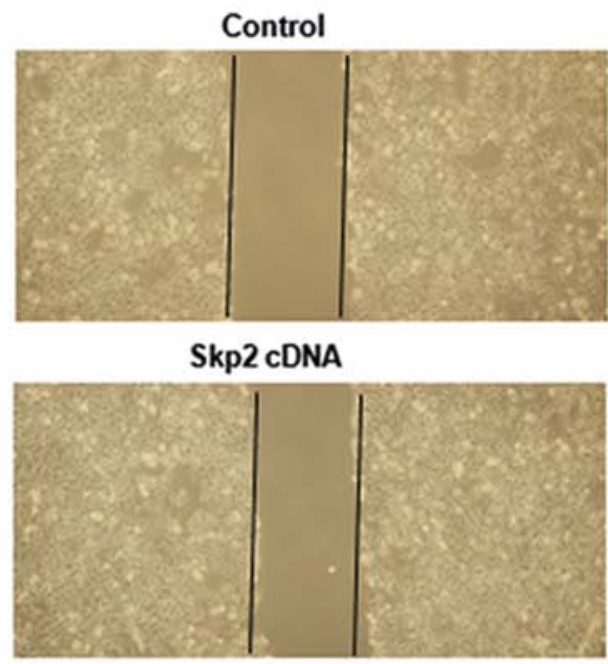

Control

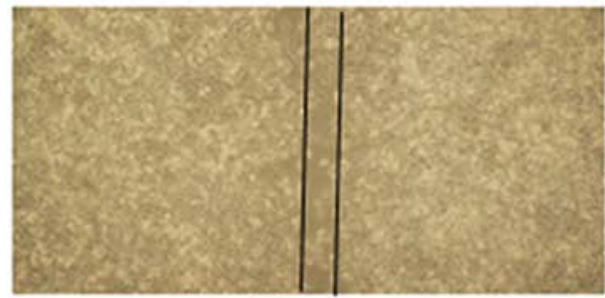

Skp2 cDNA

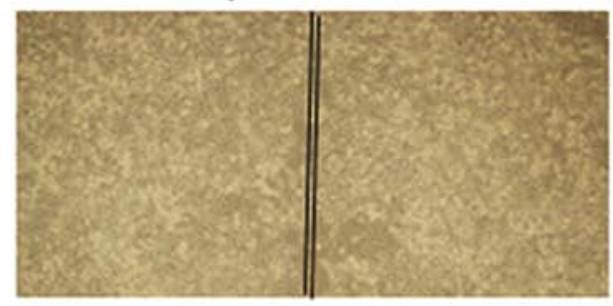

Paeoniflorin

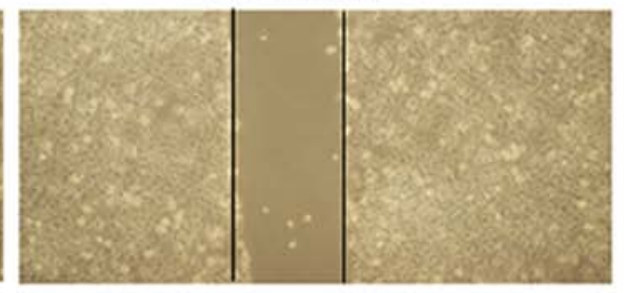

PF+Skp2 cDNA

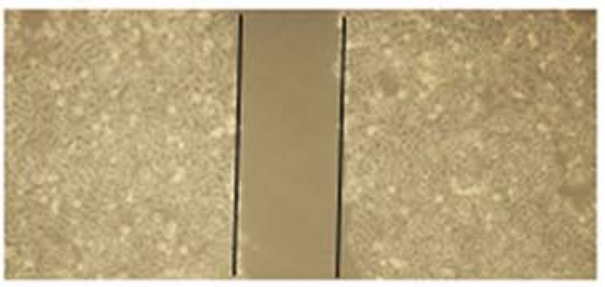

Paeoniflorin

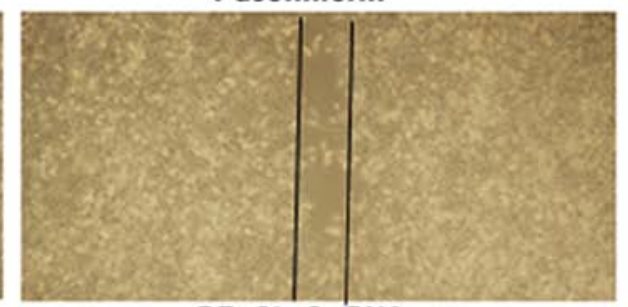

PF+Skp2 cDNA

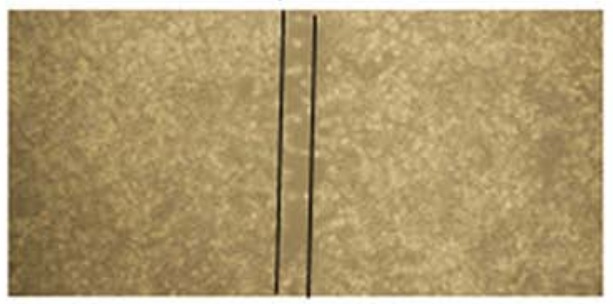

$\mathbf{O h}$

$20 \mathrm{~h}$
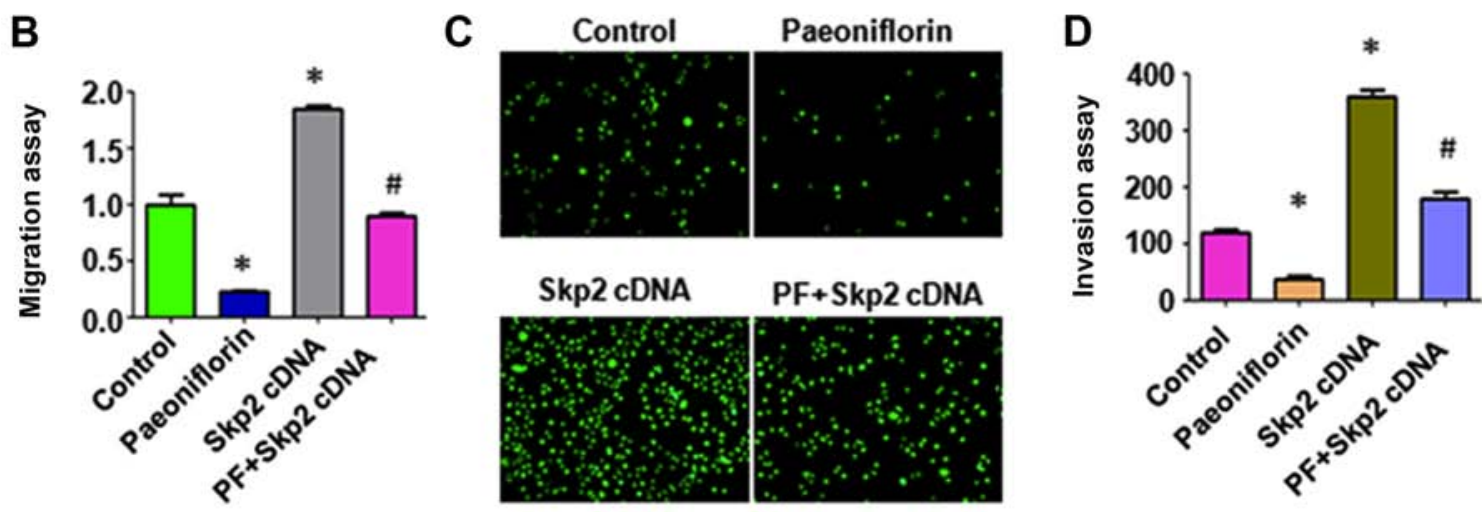

Figure 3. Overexpression of Skp2 abrogates PF-induced inhibition of migration and invasion. (A) Cell migration was evaluated by wound healing assays in HepG2 cells following Skp2 overexpression + PF treatment (magnification, $x 40)$. (B) Quantitative results of the wound healing assay. (C) Cell invasion was detected using a Transwell chamber assay in HepG2 cells following Skp2 overexpression in the presence of PF (magnification, $\mathrm{x} 100$ ). (D) Quantitative results of the Transwell chamber assay. ${ }^{*} \mathrm{P}<0.05$ vs. control; ${ }^{\prime} \mathrm{P}<0.05$ vs. PF treatment and Skp2 cDNA transfection, respectively. Skp2, S-phase kinase associated protein 2; PF, paeoniflorin.

PF-induced apoptosis in HepG2 cells (Fig. 2B). Taken together, the data demonstrated that Skp2 is primarily involved in PF-mediated cell viability inhibition and apoptosis.

Overexpression of Skp2 abolishes PF-induced inhibition of migration and invasion. The effects of Skp2 on PF-inhibited cell motility were further investigated in the liver cancer
HepG2 cells. A wound healing assay was used to measure the migration of HepG2 cells following PF treatment and Skp2 cDNA vector transfection. PF inhibited the migration of HepG2 cells (Fig. 3A and B), and overexpression of Skp2 increased cell migration in liver cancer cells and abolished PF-induced inhibition of cell migration (Fig. 3A and B). The Transwell chamber assay was used to measure the invasion of HepG2 

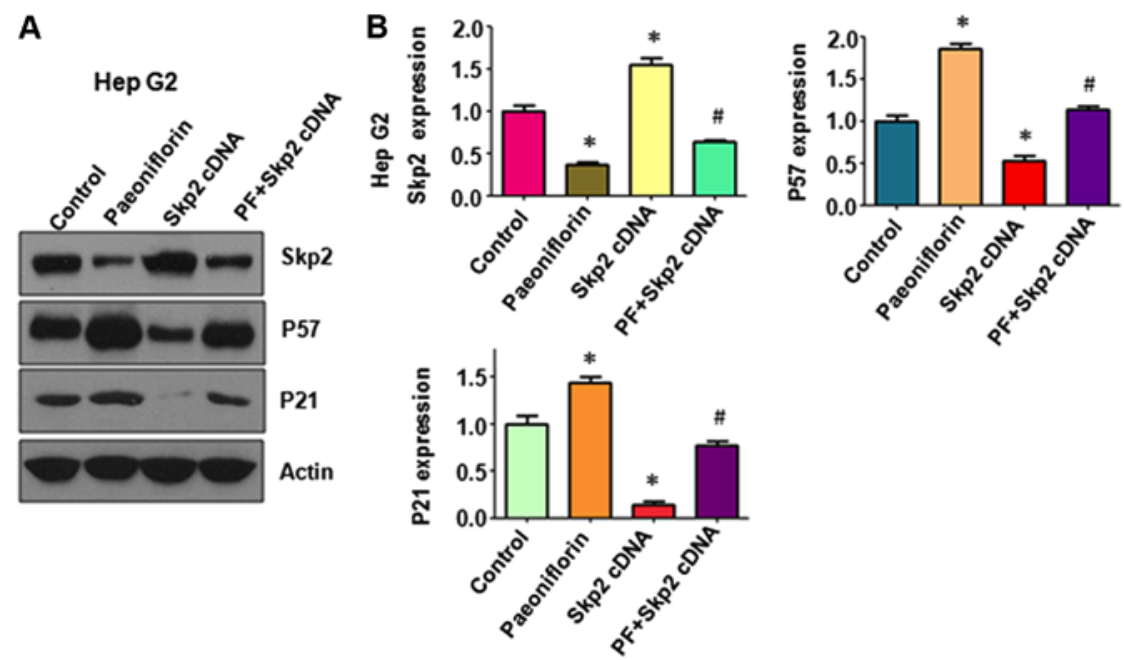

Figure 4. Overexpression of Skp2 abrogates PF-induced upregulation of p57 and p21. (A) The expression levels of Skp2 and its downstream targets, p21 and p57, were measured by western blot analysis in HepG2 cells following Skp2 overexpression in the presence of PF. (B) Densitometric analysis of the western blot bands. "P $<0.05$ vs. control; ${ }^{\#} \mathrm{P}<0.05$ vs. $\mathrm{PF}$ treatment and Skp2 cDNA transfection, respectively. Skp2, S-phase kinase associated protein 2; PF: Paeoniflorin; p21, cyclin dependent kinase inhibitor 1A; p57, cyclin dependent kinase inhibitor 1C.
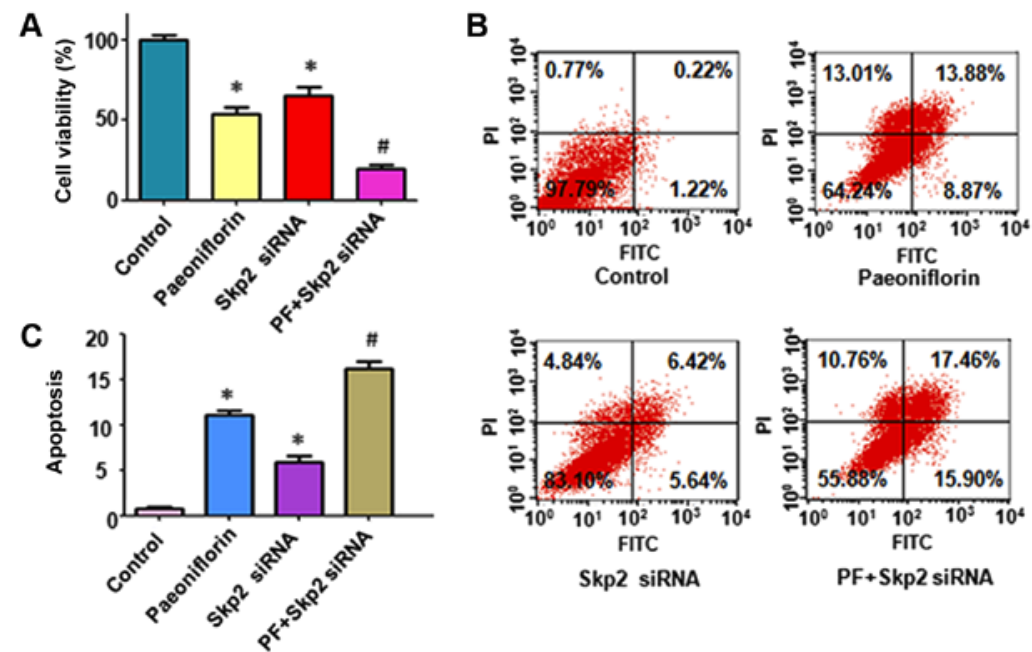

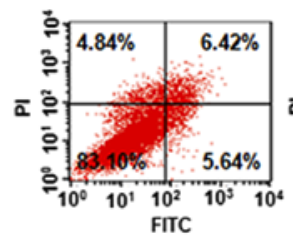

Skp2 siRNA

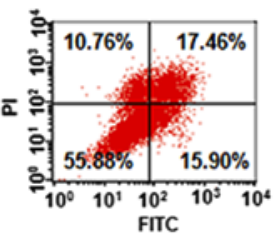

PF+Skp2 siRNA

D
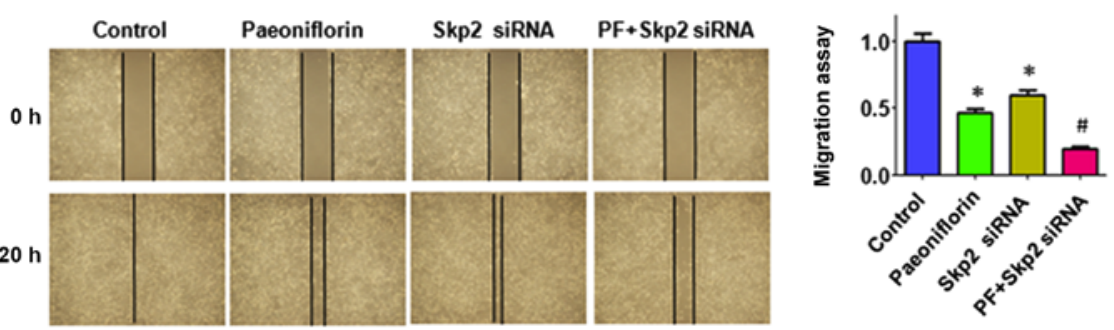

E
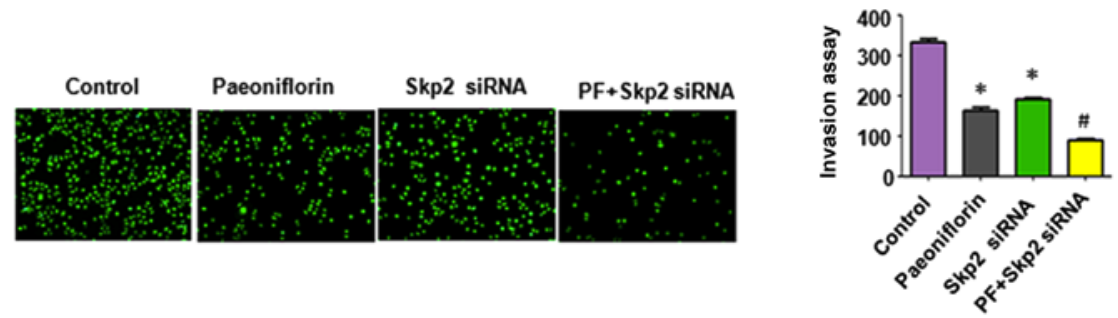

Figure 5. Downregulation of Skp2 enhances PF-induced anti-tumor activity. (A) Cell viability was measured by MTT assay following Skp2 siRNA transfection in combination with PF treatment. (B) The number of apoptotic cells was measured by flow cytometry following Skp2 siRNA transfection in combination with PF treatment. (C) Quantitative results of the flow cytometry analysis.(D) Cell migration was evaluated using a wound healing assay in HepG2 cells following Skp2 siRNA transfection in the presence of PF (magnification, $x 100$ ). The quantitative results of the wound healing assay are presented in the graph. (E) Cell invasion was examined using a Transwell chamber assay in HepG2 cells following Skp2 siRNA transfection and PF treatment (magnification, $\mathrm{x} 40$ ). The quantitative results of the Transwell chamber assay are presented in the graph. ${ }^{*} \mathrm{P}<0.05$ vs. control; ${ }^{\#} \mathrm{P}<0.05$ vs. $\mathrm{PF}$ treatment and Skp2 siRNA transfection, respectively. Skp2, S-phase kinase associated protein 2; PF, paeoniflorin; siRNA, small interfering RNA; PI, propidium iodide FITC, fluorescein isothiocyanate. 

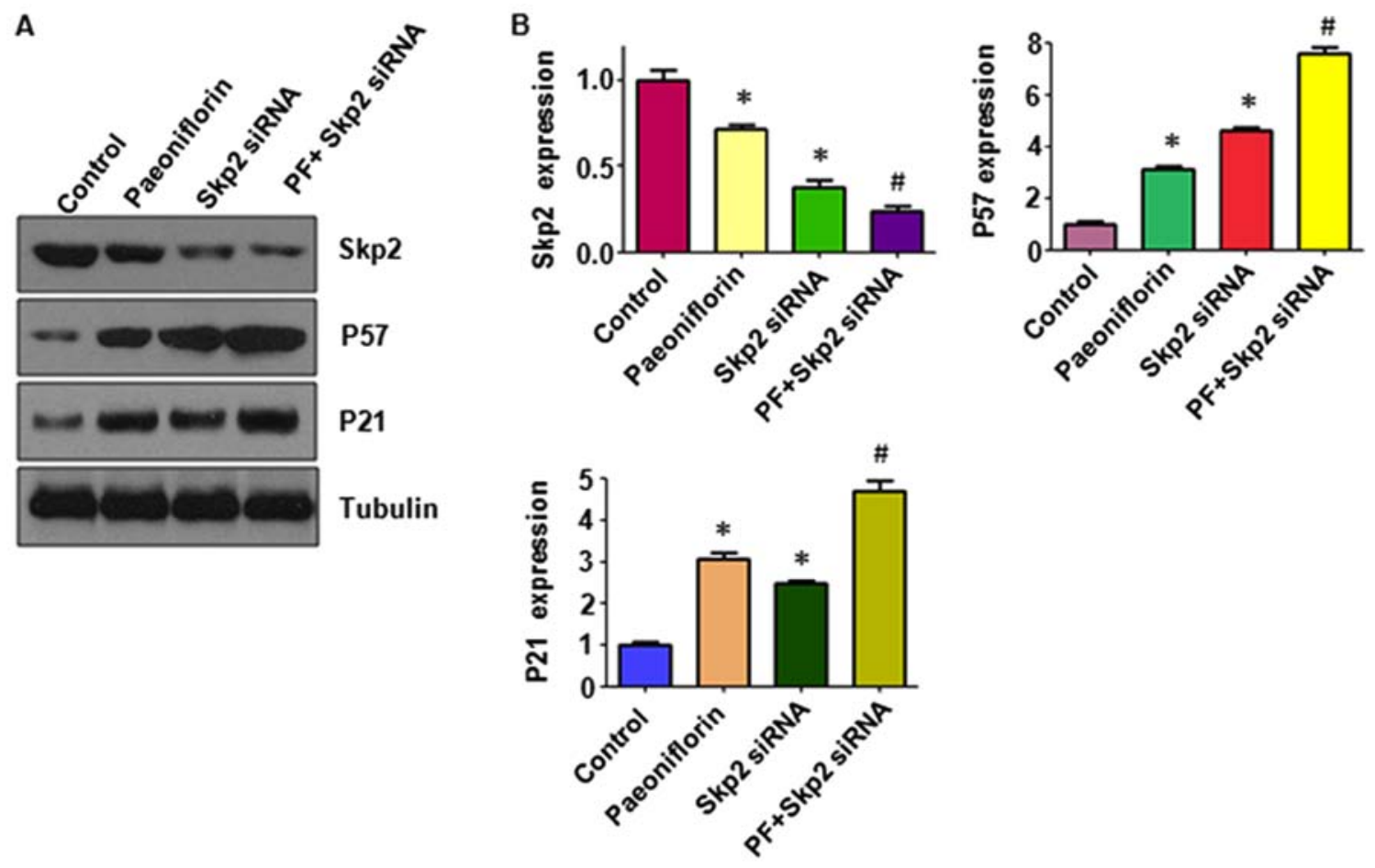

Figure 6. Downregulation of Skp2 enhances PF-induced upregulation of p57 and p21. (A) Expression levels of Skp2 and its downstream targets, p21 and p57, were measured by western blot analysis in HepG2 cells following Skp2 siRNA transfection in the presence of PF. (B) Densitometric analyses of the western blot gels. " $\mathrm{P}<0.05$ vs. control; ${ }^{\text {P }}<0.05$ vs. PF treatment and Skp2 siRNA transfection, respectively. Skp2, S-phase kinase associated protein 2; PF, paeoniflorin; p57, cyclin dependent kinase inhibitor 1C; p21, cyclin dependent kinase inhibitor 1A; siRNA, small interfering RNA.

cells following PF exposure and Skp2 cDNA transfection. The data indicated that PF inhibited invasion (Fig. 3C and D), and overexpression of Skp2 abrogated the PF-mediated inhibition of cell invasion in HepG2 cells (Fig. 3C and D). These results indicated that $\mathrm{PF}$ inhibited cell migration and invasion in part by targeting Skp2 in HepG2 cells.

Overexpression of Skp2 abolishes PF-mediated induction of p57 and p21. The effects of Skp2 overexpression on the expression of its downstream genes p57 and p21 were investigated. The western blot analysis results indicated that PF treatment inhibited Skp2 expression and increased the expression levels of p57 and p21 in HepG2 cells (Fig. 4A and B). Overexpression of Skp2 decreased the levels of p57 and p21 and abolished the PF-mediated induction of their expression in the HepG2 cells (Fig. 4A and B). Taken together, these data suggested that PF exerted its tumor suppressive function in part by the induction of p57 and p21 expression in liver cancer cells.

Downregulation of Skp2 promotes PF-induced cell viability inhibition and apoptosis. The role of Skp2 in PF-mediated cell viability inhibition was examined by Skp2 siRNA transfection in HepG2 cells, followed by PF treatment. Skp2 siRNA transfection was demonstrated to inhibit the viability of HepG2 cells (Fig. 5A). Downregulation of Skp2 in the presence of $\mathrm{PF}$ resulted in an increased rate of inhibition compared with that observed in the presence of $\mathrm{PF}$ treatment alone, or siRNA transfection alone (Fig. 5A). The induction of apoptosis was also assessed in HepG2 cells following Skp2 siRNA transfection and PF treatment. Downregulation of Skp2 induced apoptosis in HepG2 cells and enhanced PF-induced apoptosis (Fig. 5B and C). Therefore, Skp2 was determined to be an important oncoprotein involved in PF-induced cell viability inhibition and apoptosis.

Downregulation of Skp2 enhances PF-mediated cell invasion and migration. The role of Skp2 in the PF-triggered inhibition of migration was further assessed using wound healing assays in HepG2 cells treated with PF and transfected with Skp2 siRNA. Skp2 siRNA inhibited the migration of HepG2 cells (Fig. 5D). Notably, the downregulation of Skp2 promoted the PF-mediated inhibition of cell migration (Fig. 5D). Similar results were observed in HepG2 cells following Skp2 siRNA transfection in the presence of PF treatment. The Transwell chamber assay data indicated that the downregulation of Skp2 increased PF-induced cell invasion (Fig. 5E). Western blot analysis data demonstrated that the downregulation of Skp2 increased the levels of p57 and p21 in HepG2 cells (Fig. 6A and B). The downregulation of Skp2 increased PF-mediated induction of p57 and p21 expression in HepG2 cells (Fig. 6A and B). Therefore, PF was considered to exert its anti-tumor activity partly via induction of p57 and p21 expression in liver cancer cells.

\section{Discussion}

Emerging evidence has demonstrated that Skp2 serves a critical role in the development of liver cancer. For example, the nuclear expression of Skp2 predicted poor prognosis in patients with liver carcinoma (22). In addition, the inhibition of Skp2 induced apoptosis and inhibited cell proliferation in liver carcinoma cells via the upregulation of cyclin dependent 
kinase inhibitor 1B (p27) (23). It has also been suggested that Skp2 functions with N-Rasv12 or Akt to induce liver tumor development in mice (24). Recently, it was demonstrated that Skp2 promoted liver carcinoma progression via AMP-activated protein kinase-Skp2-CARM1 signaling, which led to the regulation of nutrient-deprived autophagy induction (8). Notably, the Hippo signaling pathway can suppress liver tumorigenesis via the Skp2 pathway (25). Skp2 and cyclin-dependent kinases regulatory subunit 1 promoted the degradation of cell cycle regulators and were associated with liver carcinoma prognosis (26). The X protein of hepatitis B virus (HBV) binds to Skp2 and inhibits the ubiquitination and proteasomal degradation of c-Myc in human hepatoma cell lines (27). Similarly, HBV core promoter mutations enhanced cell proliferation via transcription factor E2F1-mediated upregulation of Skp2 transcription $(28,29)$. Hepatocyte growth factor suppressed HepG 2 cell proliferation by increasing p27 expression and by decreasing Skp2 expression (30). These studies have suggested that Skp2 is an important oncoprotein participating in liver cancer development, and that the inhibition of Skp2 may be used as a strategy to treat liver cancer.

PF has been demonstrated to inhibit the growth of HepG2 and Bel-7402 cells, and to decrease the invasion, adhesion and metastasis of liver cancer cells (14). In concordance with these data, the present study demonstrated that PF suppressed cell viability, and inhibited the migration and invasion of liver cancer HepG2 cells. A previous study indicated that PF induced apoptosis in liver cancer HepG2 and SMMC-7721 cells by the inhibition of Bcl-2 and the induction of Bax and cleaved caspase- 3 expression, and the downregulation of the prostaglandin E receptor EP2 subtype in liver cancer cells (31). PF exposure decreased the expression levels of matrix metalloprotein-9 (MMP-9) and extracellular signal-regulated kinase, whereas it increased the levels of E-cadherin in liver cancer cells (14). The data indicated that PF inhibited the expression levels of Skp2 in liver cancer cells. Recently, it was suggested that PF exerted antitumor effects by inactivating Skp2 in glioma cells (32). This suggested that PF may decrease the expression levels of Skp2 in various types of human cancer, indicating that PF may be a potential inhibitor of Skp2 in multiple cancer types.

Several agents have been identified to inhibit the expression of Skp2 in human cancer. For example, troglitazone, an agonist of peroxisome proliferator-activated receptor gamma, was identified to downregulate the expression levels of Skp2 and to induce p27-associated cell cycle arrest in liver cancer cells $(33,34)$. EB1089 induced Skp2-dependent p27 accumulation, resulting in inhibition of cell growth and cell cycle G1 phase arrest in hepatoma cells (35). Longikaurin A, a natural kauranoid diterpenoid, induced cell cycle arrest by inhibition of Skp2 expression in liver carcinoma cells (36). Simvastatin, an HMG-Co-enzyme A reductase inhibitor, induced cell cycle arrest via suppression of the STAT3/Skp2 axis in order to promote p27 and p21 accumulation in liver carcinoma cells (37). Curcumin has been demonstrated to inhibit the expression of Skp2 in several human cancer types $(17,38,39)$. Rottlerin exhibited its anti-tumor activity by the inhibition of Skp2 in breast cancer and pancreatic cancer cells $(18,40)$. The present study demonstrated that PF inhibited Skp2 expression in liver cancer cells. It is important to note that the present study had certain limitations, such as the use of only 1 cell line. Future studies are required to determine the role of PF in multiple cell lines. Regarding wound healing assays, serum-starvation is applied to halt proliferation and ensure that the wound closure is a direct result of migration. However, the present study used $10 \%$ FBS in the wound healing assay as HepG2 cell viability is notably decreased if cells are serum starved (41). Future studies should also examine whether PF regulated the cell cycle via inhibition of Skp2. A previous study reported that PF did not affect normal hepatocyte growth in mice (16); however, whether PF affects normal and healthy liver cells in human requires further elucidation.

\section{Acknowledgements}

Not applicable.

\section{Funding}

No funding was received.

\section{Availability of data and materials}

All data generated or analyzed during this study are included in this published article.

\section{Authors' contributions}

HL and LL designed the present study. HL, LZ, JZ and ZW acquired and analyzed the data. LL supervised the present study, and HL, LZ and LL drafted and edited the initial manuscript. All authors read and approved the final manuscript.

\section{Ethics approval and consent to participate}

Not applicable.

\section{Patient consent for publication}

Not applicable.

\section{Competing interests}

The authors declare that they have no competing interests.

\section{References}

1. Siegel RL, Miller KD and Jemal A: Cancer statistics, 2018. CA Cancer J Clin 68: 7-30, 2018.

2. Jemal A, Ward EM, Johnson CJ, Cronin KA, Ma J, Ryerson B, Mariotto A, Lake AJ, Wilson R, Sherman RL, et al: Annual Report to the Nation on the Status of Cancer, 1975-2014, Featuring Survival. J Natl Cancer Inst 109, 2017.

3. Wang G, Chan CH, Gao Y and Lin HK: Novel roles of Skp2 E3 ligase in cellular senescence, cancer progression, and metastasis. Chin J Cancer 31: 169-177, 2012.

4. Frescas D and Pagano M: Deregulated proteolysis by the F-box proteins SKP2 and beta-TrCP: Tipping the scales of cancer. Nat Rev Cancer 8: 438-449, 2008.

5. Wang Z, Liu P, Inuzuka $\mathrm{H}$ and Wei W: Roles of F-box proteins in cancer. Nat Rev Cancer 14: 233-247, 2014.

6. Chan CH, Morrow JK, Zhang S and Lin HK: Skp2: A dream target in the coming age of cancer therapy. Cell Cycle 13: 679-680, 2014. 
7. Lu M, Ma J, Xue W, Cheng C, Wang Y, Zhao Y, Ke Q, Liu H, Liu Y, Li P, et al: The expression and prognosis of FOXO3a and Skp2 in human hepatocellular carcinoma. Pathol Oncol Res 15: 679-687, 2009

8. Wei X, Li X, Yan W, Zhang X, Sun Y and Zhang F: SKP2 promotes hepatocellular carcinoma progression through nuclear AMPK-SKP2-CARM1 signaling transcriptionally regulating nutrient-deprived autophagy induction. Cell Physiol Biochem 47: 2484-2497, 2018.

9. Chen A, Wang H, Zhang Y, Wang X, Yu L, Xu W, Xu W and Lin Y: Paeoniflorin exerts neuroprotective effects against glutamateinduced PC12 cellular cytotoxicity by inhibiting apoptosis. Int J Mol Med 40: 825-833, 2017.

10. Gu P, Zhu L, Liu Y, Zhang L, Liu J and Shen H: Protective effects of paeoniflorin on TNBS-induced ulcerative colitis through inhibiting NF-kappaB pathway and apoptosis in mice. Int Immunopharmacol 50: 152-160, 2017.

11. Kapoor S: The emerging antineoplastic effects of paeoniflorin. Anticancer Drugs 24: 429, 2013.

12. Hu Z, Qin F, Gao S, Zhen Y, Huang D and Dong L: Paeoniflorin exerts protective effect on radiation-induced hepatic fibrosis in rats via TGF- $\beta 1 /$ Smads signaling pathway. Am J Transl Res 10: 1012-1021, 2018.

13. Li JZ, Tang XN, Li TT, Liu LJ, Yu SY,Zhou GY, Shao QR, Sun HP, Wu C and Yang Y: Paeoniflorin inhibits doxorubicin-induced cardiomyocyte apoptosis by downregulating microRNA-1 expression. Exp Ther Med 11: 2407-2412, 2016.

14. Lu JT, He W, Song SS and Wei W: Paeoniflorin inhibited the tumor invasion and metastasis in human hepatocellular carcinoma cells. Bratisl Lek Listy 115: 427-433, 2014

15. Wang H, Zhou H, Wang CX, Li YS, Xie HY, Luo JD and Zhou Y: Paeoniflorin inhibits growth of human colorectal carcinoma HT 29 cells in vitro and in vivo. Food Chem Toxicol 50: 1560-1567, 2012

16. Jiang Z, Chen W, Yan X, Bi L, Guo S and Zhan Z: Paeoniflorin protects cells from GalN/TNF- $\alpha$-induced apoptosis via ER stress and mitochondria-dependent pathways in human L02 hepatocytes. Acta Biochim Biophys Sin (Shanghai) 46: 357-367, 2014

17. Wang L, Ye X, Cai X, Su J, Ma R, Yin X, Zhou X, Li H and Wang Z: Curcumin suppresses cell growth and invasion and induces apoptosis by down-regulation of Skp2 pathway in glioma cells. Oncotarget 6: 18027-18037, 2015.

18. Yin X, Zhang Y, Su J, Hou Y, Wang L, Ye X, Zhao Z, Zhou X, Li Y and Wang Z: Rottlerin exerts its anti-tumor activity through inhibition of Skp2 in breast cancer cells. Oncotarget 7: 66512-66524, 2016

19. Livak KJ and Schmittgen TD: Analysis of relative gene expression data using real-time quantitative PCR and the 2(-Delta Delta C(T)) method. Methods 25: 402-408, 2001

20. Huang $\mathrm{Y}$, Zhao M, Xu H, Wang K, Fu Z, Jiang $\mathrm{Y}$ and Yao Z: RASAL2 down-regulation in ovarian cancer promotes epithelial-mesenchymal transition and metastasis. Oncotarget 5 : 6734-6745, 2014

21. Wang L, Hou Y, Yin X, Su J, Zhao Z, Ye X, Zhou X, Zhou L and Wang Z: Rottlerin inhibits cell growth and invasion via down-regulation of $\mathrm{Cdc} 20$ in glioma cells. Oncotarget 7 : 69770-69782, 2016

22. Shin E, Kim SH, Jeong HY, Jang JJ and Lee K: Nuclear expression of S-phase kinase-associated protein 2 predicts poor prognosis of hepatocellular carcinoma. Apmis 120: 349-357, 2012.

23. Qi M, Liu D, Zhang S, Hu P and Sang T: Inhibition of S-phase kinase-associated protein 2-mediated p27 degradation suppresses tumorigenesis and the progression of hepatocellular carcinoma. Mol Med Rep 11: 3934-3940, 2015.

24. Delogu S, Wang C, Cigliano A, Utpatel K, Sini M, Longerich T, Waldburger N, Breuhahn K, Jiang L, Ribback S, et al: SKP2 cooperates with N-Ras or AKT to induce liver tumor development in mice. Oncotarget 6: 2222-2234, 2015.

25. Zhang S, Chen Q, Liu Q, Li Y, Sun X, Hong L, Ji S, Liu C, Geng J, Zhang W, et al: Hippo signaling suppresses cell ploidy and tumorigenesis through Skp2. Cancer Cell 31: 669-684,e7, 2017.

26. Calvisi DF, Ladu S, Pinna F, Frau M, Tomasi ML, Sini M, Simile MM, Bonelli P, Muroni MR, Seddaiu MA, et al: SKP2 and CKS1 promote degradation of cell cycle regulators and are associated with hepatocellular carcinoma prognosis. Gastroenterology 137: 1816-1826,e1-10, 2009.
27. Kalra $\mathrm{N}$ and Kumar V: The $\mathrm{X}$ protein of hepatitis $\mathrm{B}$ virus binds to the $\mathrm{F}$ box protein Skp2 and inhibits the ubiquitination and proteasomal degradation of c-Myc. FEBS Lett 580: 431-436, 2006.

28. Huang Y, Tai AW, Tong S and Lok AS: HBV core promoter mutations promote cellular proliferation through E2F1-mediated upregulation of S-phase kinase-associated protein 2 transcription. J Hepatol 58: 1068-1073, 2013.

29. Huang Y, Tong S, Tai AW, Hussain M and Lok AS: Hepatitis B virus core promoter mutations contribute to hepatocarcinogenesis by deregulating SKP2 and its target, p21. Gastroenterology 141: 1412-1421, e1-e5, 2011.

30. Zhang H, Ozaki I, Mizuta T, Yoshimura T, Matsuhashi S, Hisatomi A, Tadano J, Sakai T and Yamamoto K: Mechanism of beta 1-integrin-mediated hepatoma cell growth involves p27 and S-phase kinase-associated protein 2. Hepatology 38: 305-313, 2003.

31. Hu S, Sun W, Wei W, Wang D, Jin J, Wu J, Chen J, Wu H and Wang Q: Involvement of the prostaglandin E receptor EP2 in paeoniflorin-induced human hepatoma cell apoptosis. Anticancer Drugs 24: 140-149, 2013.

32. Ouyang J, Xu H, Li M, Dai X, Fu F, Zhang X and Lan Q: Paeoniflorin exerts antitumor effects by inactivating $S$ phase kinase-associated protein 2 in glioma cells. Oncol Rep 39: 1052-1062, 2018

33. Zhong WB, Tsai YC, Chin LH, Tseng JH, Tang LW, Horng S, Fan YC and Hsu SP: A synergistic anti-cancer effect of troglitazone and lovastatin in a human anaplastic thyroid cancer cell line and in a mouse xenograft model. Int J Mol Sci 19: pii: E1834, 2018.

34. Motomura W, Takahashi N, Nagamine M, Sawamukai M, Tanno S, Kohgo Y and Okumura T: Growth arrest by troglitazone is mediated by $\mathrm{p} 27 \mathrm{Kip} 1$ accumulation, which results from dual inhibition of proteasome activity and Skp2 expression in human hepatocellular carcinoma cells. Int J Cancer 108: 41-46, 2004.

35. Luo W, Chen Y, Liu M, Du K, Zheng G, Cai T, Zhang W, Zhao F, Yao T, Yang R and Chen J: EB1089 induces Skp2-dependent p27 accumulation, leading to cell growth inhibition and cell cycle G1 phase arrest in human hepatoma cells. Cancer Invest 27: 29-37, 2009.

36. Liao YJ, Bai HY, Li ZH, Zou J, Chen JW, Zheng F, Zhang JX Mai SJ, Zeng MS, Sun HD, et al: Longikaurin A, a natural ent-kaurane, induces $\mathrm{G} 2 / \mathrm{M}$ phase arrest via downregulation of Skp2 and apoptosis induction through ROS/JNK/c-Jun pathway in hepatocellular carcinoma cells. Cell Death Dis 5: e1137, 2014.

37. Wang ST,HoHJ,Lin JT, Shieh JJ and Wu CY: Simvastatin-induced cell cycle arrest through inhibition of STAT3/SKP2 axis and activation of AMPK to promote $\mathrm{p} 27$ and p21 accumulation in hepatocellular carcinoma cells. Cell Death Dis 8: e2626, 2017.

38. Feng S, Wang Y, Zhang R, Yang G, Liang Z, Wang Z and Zhang G: Curcumin exerts its antitumor activity through regulation of $\mathrm{miR}-7 / \mathrm{Skp} 2 / \mathrm{p} 21$ in nasopharyngeal carcinoma cells. Onco Targets Ther 10: 2377-2388, 2017.

39. Su J, Zhou X, Wang L, Yin X and Wang Z: Curcumin inhibits cell growth and invasion and induces apoptosis through down-regulation of Skp2 in pancreatic cancer cells. Am J Cancer Res 6: 1949-1962, 2016.

40. Su J, Wang L, Yin X, Zhao Z, Hou Y, Ye X, Zhou X and Wang Z: Rottlerin exhibits anti-cancer effect through inactivation of $S$ phase kinase-associated protein 2 in pancreatic cancer cells. Am J Cancer Res 6: 2178-2191, 2016.

41. Dituri F, Mazzocca A, Fernando J, Peidrò FJ, Papappicco P, Fabregat I, De Santis F, Paradiso A, Sabbà C and Giannelli G: Differential inhibition of the TGF- $\beta$ signaling pathway in HCC cells using the small molecule inhibitor LY2157299 and the D10 monoclonal antibody against TGF- $\beta$ receptor Type II. PLoS One 8: e67109, 2013.

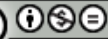

This work is licensed under a Creative Commons Attribution-NonCommercial-NoDerivatives 4.0 International (CC BY-NC-ND 4.0) License. 